\title{
Post-processing of solutions of incompressible Navier-Stokes equations on rotating spheres
}

\author{
$\begin{array}{ll}\text { M. Ganesh } & \text { Q. T. Le Gia } \\ & \end{array}$
}

(Received 14 August 2008; revised 03 October 2008)

\begin{abstract}
We describe a post-processing technique (requiring only solutions of linear stationary problems) to improve the resolution of Galerkin solutions of the time dependent nonlinear incompressible Navier-Stokes equations on the rotating unit sphere. Numerical experiments illustrate the advantage of this more efficient method to simulate higher modes to approximate the divergence-free velocity field.
\end{abstract}

\section{Contents} 1446-8735. (Print two pages per sheet of paper.) 
3 A post-processing pseudospectral method

4 Numerical results

C98

References

C105

\section{Introduction}

The pseudospectral Galerkin method is a standard approach for computer modelling of the large scale atmospheric dynamics through the Navier-Stokes equations (NSE) on the rotating Earth, see for example the recent work by Fengler and Freeden [3] and references therein. The computable pseudospectral solution with $\mathrm{N}$ modes, approximating the unknown tangential surface divergence free velocity field of the NSE, is of the form

$$
\mathbf{u}_{N}=\sum_{n=1}^{N} \sum_{|m| \leq n} \alpha_{n, m}(t) \mathbf{Z}_{n, m},
$$

where $\mathbf{Z}_{n, m}$ are the tangential surface divergence free eigenfunctions of the Stokes operator on the chosen manifold, approximating the shape of the Earth. The rotating manifold was chosen to be the unit sphere by Fengler and Freeden [3]. (A standard surface model to study the atmospheric circulation on large planets is the sphere.) The number of time dependent unknowns in (1) is $\mathbf{N}^{2}+2 \mathrm{~N}$ and in pseudospectral methods these are obtained by first projecting the spatial part of the NSE into the approximation space spanned by the $\mathrm{N}^{2}+2 \mathrm{~N}$ eigenfunctions, leading to the Galerkin (Fourier coefficient) equations, and then simulating the resulting $\left(\mathrm{N}^{2}+2 \mathrm{~N}\right)$-dimensional nonlinear stiff ordinary differential systems in time. At each small time step of the evolution process the computational cost is dominated by setting up of the quadratic nonlinearity in the projected NSE with $\mathrm{O}\left(\mathrm{N}^{4}\right)$ terms. Recall that each Fourier coefficient requires integration on the sphere and approximation by quadratures which substantially increases the computational complexity. 
In order to obtain a fine resolution in the approximate velocity field, the value of $\mathbf{N}$ in (1) needs to be high. In the case of three dimensional timedependent nonlinear partial differential equations, due to $\mathrm{O}\left(\mathrm{N}^{4}\right)$ complexity in setting up the nonlinear equations at each time step and evolving over a long period of time, it is desirable to simulate with as small a value of $\mathrm{N}$ as possible and yet obtain accurate velocity and pressure fields.

In particular, for the NSE on periodic domains, after solving appropriate nonlinear time dependent systems only for low frequency approximations, one may obtain some of the remaining modes using approximate inertial manifolds (Marion and Temam [8]). The exact inertial manifold is an operator that determines the high frequency modes as a function of the computed low frequency modes.

There are several ways to approximate the exact inertial manifold. For example one may restrict to $2 \mathrm{~N}$ modes and then, based on the physical behaviour of the velocity field, use a linearised version of the NSE. One such well known approximate inertial manifold method, leading to higher modes in the velocity field from low frequency modes, is called the nonlinear Galerkin method, introduced by Marion and Temam [8] for the NSE on bounded two dimensional domains with the nonslip boundary condition or on the plane $\mathbb{R}^{2}$ with periodic boundary conditions. For the rotating sphere model, Fengler and Freeden [3] used the nonlinear Galerkin method to obtain approximate velocity fields with $2 \mathrm{~N}$ modes.

The nonlinear Galerkin method has the advantage of reducing the nonlinearity in the evolution system of the standard Galerkin approximations. However, for each small positive evolution time step, to obtain the low frequency nonlinear Galerkin solutions, the corresponding high frequency modes should be computed as well. In particular, the main disadvantage of the nonlinear Galerkin method [3] is that for computing the $2 \mathrm{~N}$ modes, a $4\left(\mathrm{~N}^{2}+\mathrm{N}\right)$ dimensional coupled system of differential equations needs to be simulated, with the first $\left(\mathrm{N}^{2}+2 \mathrm{~N}\right)$-dimensional equation being nonlinear and the rest being linear elliptic problems. 
An ideal approach would be to first compute low frequency approximations, say $\mathbf{u}_{\mathrm{N}}(\mathrm{t})$, for all $t \in[0, \mathrm{~T}]$ and then, for any particular desired time $t^{*}$, obtain high-resolution modes by solving just one simple stationary equation using $\mathbf{u}_{\mathrm{N}}\left(\mathbf{t}^{*}\right)$. Such a method, known as the post-processing technique, was introduced by Archilla et al. [1] as a novel approach to approximate inertial manifolds. This method was applied for some parabolic problems on domains and yielded solutions with accuracy similar to the nonlinear Galerkin solutions.

This article develops a post-processing version of the recent work [5] on the pseudospectral quadrature Galerkin method for the NSE on rotating spheres. We recall the weak form of the NSE in the next section and describe the post-processing approach in Section 3. Numerical experiments in Section 4 for a known velocity field with $2 \mathrm{~N}$ Fourier modes and a benchmark velocity flow generated by a random initial velocity field with Reynolds number of the order $10^{4}$ highlight the efficiency of this post-processing approach.

\section{The NSE on the rotating surface}

We are interested in simulating a tangential velocity flow on the unit sphere $S$, denoted by $\mathbf{u}=\mathbf{u}(\hat{\mathbf{x}}, \mathrm{t})=\left(\mathrm{u}_{1}(\hat{\mathbf{x}}, \mathrm{t}), \mathrm{u}_{2}(\hat{\mathbf{x}}, \mathrm{t}), \mathrm{u}_{3}(\hat{\mathbf{x}}, \mathrm{t})\right)^{\mathrm{T}}, \hat{\mathbf{x}} \in \mathrm{S}$ and $\mathrm{t} \in[0, T]$, with pressure $p=p(\hat{\mathbf{x}}, t)$, satisfying the the incompressible NSE $[3,6,7,9]$

$$
\begin{aligned}
\frac{\partial}{\partial t} \mathbf{u}+\nabla_{\mathbf{u}} \mathbf{u}-v \Delta \mathbf{u}+\boldsymbol{w} \times \mathbf{u}+\frac{1}{\rho} \operatorname{Grad} p & =\mathbf{f} \text { on } S, \\
\left.\mathbf{u}\right|_{\mathbf{t}=0}=\mathbf{u}_{0}, \quad \operatorname{Div} \mathbf{u}=0 & \text { on } S,
\end{aligned}
$$

where the velocity flow is driven by the external field $\mathbf{f}=\mathbf{f}(\hat{\mathbf{x}}, t)$, the rotation effect is included in the normal vector field Coriolis term $\boldsymbol{\omega}(\hat{\mathbf{x}})=2 \hat{\mathbf{x}} \Omega \cos \theta$, with constant rotation rate $\Omega, \cos \theta$ being the vertical third component of 
$\hat{\mathbf{x}} \in \mathbf{S}$ and $\theta$ is the latitudinal variable. In (2), $\boldsymbol{v}$ and $\rho$ are respectively the constant viscosity and density of the fluid.

Note that all spatial differential operators in (2)-(3) are defined on the surface $S$. Among these, the surface divergence and gradient, denoted respectively by Div, Grad, are well known [6, 7], and the vector Laplace-Beltrami operator $-\Delta$ satisfies

$$
-\Delta \mathbf{v}=\operatorname{Curl~Curl}_{\widehat{\mathbf{x}}} \mathbf{v}-\operatorname{Grad} \operatorname{Div} \mathbf{v}
$$

where the rate of rotation of a scalar function $v$, a normal vector field $\mathbf{w}=$ $w \hat{\mathbf{x}}$, and a tangential vector field $\mathbf{v}$ on $\mathbf{S}$ are respectively defined by

$\operatorname{Curl} v=-\hat{\mathbf{x}} \times \operatorname{Grad} v, \quad \operatorname{Curl} \mathbf{w}=-\hat{\mathbf{x}} \times \operatorname{Grad} w, \quad \operatorname{Curl}_{\hat{\mathbf{x}}} \mathbf{v}=-\hat{\mathbf{x}} \operatorname{Div}(\hat{\mathbf{x}} \times \mathbf{v})$. In (2), the nonlinear term $\nabla_{\mathbf{u}} \mathbf{u}$ is the covariant derivative

$$
\begin{aligned}
2 \nabla_{\mathbf{w}} \mathbf{v}= & -\operatorname{Curl}(\mathbf{w} \times \mathbf{v})+\operatorname{Grad}(\mathbf{w} \cdot \mathbf{v})-\mathbf{v} \operatorname{Div} \mathbf{w}+\mathbf{w} \operatorname{Div} \mathbf{v} \\
& -\mathbf{v} \times \operatorname{Curl}_{\widehat{\mathbf{x}}} \mathbf{w}-\mathbf{w} \times \operatorname{Curl}_{\widehat{\mathbf{x}}} \mathbf{v} .
\end{aligned}
$$

Restriction of the vector Laplace-Beltrami operator in (4) to the tangential divergence free functions reduces to the Stokes operator

$$
\boldsymbol{A}=\operatorname{Curl~Curl}_{\widehat{\mathbf{x}}}=-\Delta \mathrm{P}_{\mathrm{Curl}},
$$

where $P_{\text {Curl }}$ is the projection onto the space of smooth tangential divergence free functions, denoted throughout by $\mathrm{V}$. The infinite dimensional space $\mathrm{V}$ is spanned by all polynomial eigenfunctions of the Stokes operator of degree $n=1,2, \ldots$ For a fixed degree $n$, there are $2 n+1$ degree $n$ orthonormal eigenfunctions, denoted throughout by $\mathbf{Z}_{n, m}, \mathfrak{m}=-\mathfrak{n}, \ldots, n$. Here the orthonormality is with respect to the $\mathrm{L}^{2}$-inner product $(\cdot, \cdot)$ for vector fields (and the associated $\mathrm{L}^{2}$-norm is denoted throughout by $\|\cdot\|$ ).

The next step is to remove the pressure term Gradp in (2). This is achieved by a weak formulation. That is, multiply (2) by functions in $V$ and integrate using the Gauss surface divergence theorem to remove Grad p:

$$
(\operatorname{Grad} p, \mathbf{w}):=\int_{S} \operatorname{Grad} p \cdot \overline{\mathbf{w}} \mathrm{d} S=-\int_{S} p \cdot \operatorname{Div} \overline{\mathbf{w}} \mathrm{d} S=0, \quad \mathbf{w} \in \mathrm{V} .
$$


For the nonlinear term in the weak formulation of (2), we consider

$$
\mathbf{b}(\mathbf{v}, \mathbf{w}, \overline{\mathbf{z}})=\left(\nabla_{\mathbf{v}} \mathbf{w}, \overline{\mathbf{z}}\right)=\int_{S} \nabla_{\mathbf{v}} \mathbf{w} \cdot \mathbf{z} \mathrm{d} S, \quad \mathbf{v}, \mathbf{w}, \mathbf{z} \in \mathrm{V} .
$$

The Coriolis operator on $\mathrm{V}$ is defined by

$$
(\mathbf{C v})(\widehat{\mathbf{x}})=\boldsymbol{\omega}(\widehat{\mathbf{x}}) \times \mathbf{v}(\widehat{\mathbf{x}})=\omega(\widehat{\mathbf{x}})(\hat{\mathbf{x}} \times \mathbf{v}), \quad \boldsymbol{\omega}(\widehat{\mathbf{x}})=2 \Omega \cos \theta,
$$

for $\mathbf{v} \in \mathbf{V}$ and $\hat{\mathbf{x}} \in \mathbf{S}$. Thus, a weak form of the NSE (2)-(3) is

$$
\frac{\partial}{\partial t}(\mathbf{u}, \mathbf{v})+\mathrm{b}(\mathbf{u}, \mathbf{u}, \mathbf{v})+v\left(\operatorname{Curl}_{\widehat{\mathbf{x}}} \mathbf{u}, \operatorname{Curl}_{\widehat{\mathbf{x}}} \mathbf{v}\right)+(\mathbf{C u}, \mathbf{v})=(\mathbf{f}, \mathbf{v}), \quad \mathbf{v} \in \mathbf{V},
$$

or in operator form with $(\mathbf{B}(\mathbf{u}, \mathbf{u}), \mathbf{v})=\mathbf{b}(\mathbf{u}, \mathbf{u}, \mathbf{v})$, (9) is written as

$$
\frac{\partial}{\partial t} \mathbf{u}+v \mathbf{A} \mathbf{u}+\mathbf{B}(\mathbf{u}, \mathbf{u})+\mathbf{C} \mathbf{u}=\mathbf{f}, \quad \mathbf{u}(0)=\mathbf{u}_{0} .
$$

\section{$3 \quad$ A post-processing pseudospectral method}

The pseudospectral quadrature Galerkin method for the NSE was developed and analysed in detail by Ganesh et al. [5] with approximate velocity in

$$
V_{N}:=\operatorname{span}\left\{Z_{n, m}: n=1, \ldots, N, m=-n, \ldots, n\right\} .
$$

Let $\Pi_{N}$ be the orthogonal projection from $V$ onto $V_{N}$ and let $Q_{N}=I-\Pi_{N}$. The solution of (9) is decomposed uniquely as

$$
\mathbf{u}=\mathbf{p}_{\mathrm{N}}+\mathbf{q}_{\mathrm{N}}, \quad \mathbf{p}_{\mathrm{N}}=\Pi_{\mathrm{N}} \mathbf{u}, \quad \mathbf{q}_{\mathrm{N}}=\mathrm{Q}_{\mathrm{N}} \mathbf{u} .
$$

Details described by Ganesh et al. [5] lead to computation and analysis of

$$
\mathbf{u}_{N}(t):=\sum_{n=1}^{N} \sum_{|m| \leq n} \alpha_{n, m}(t) \mathbf{Z}_{n, m}
$$




$$
\frac{d \mathbf{u}_{N}}{d t}+\Pi_{N} \mathbf{B}\left(\mathbf{u}_{N}, \mathbf{u}_{N}\right)+v A \mathbf{u}_{N}+\mathbf{C} \mathbf{u}_{N}=\Pi_{N} \mathbf{f}, \quad \mathbf{u}_{N}(0)=\Pi_{N} \mathbf{u}_{0},(13)
$$

where we used that $\boldsymbol{A}$ and $\mathbf{C}$ map $\mathbf{V}_{\mathrm{N}}$ to $\mathbf{V}_{\mathrm{N}}$ because $\mathbf{Z}_{n, m}$ are eigenfunctions of $\boldsymbol{A}$, for $n=1, \ldots, N, m=-n, \ldots, n$, and $\left(\mathbf{C Z}_{n, m}, \mathbf{Z}_{j, k}\right)=0$ if $n \neq j$ or $\mathrm{m} \neq \mathrm{k}$, for $\mathrm{j}=1,2, \ldots$ and $\mathrm{k}=-\mathrm{j}, \ldots, \mathrm{j}$.

The system (13) has $\mathrm{N}^{2}+2 \mathrm{~N}$ coupled ordinary differential equations for the unknown coefficients $\alpha_{n, m}(t)$ in (12). Error analysis by Ganesh et al. [5] gives a spectrally accurate upper bound for $\left\|\mathbf{u}-\mathbf{u}_{\mathrm{N}}\right\|$. For a lower bound, note that the Galerkin error $\mathbf{u}-\mathbf{u}_{\mathrm{N}}$ can never be smaller than the best-approximation error $\mathbf{u}-\mathbf{p}_{\mathrm{N}}=\mathbf{q}_{\mathrm{N}}$, that is

$$
\left\|\mathbf{u}-\mathbf{u}_{\mathrm{N}}\right\| \geq\left\|\mathbf{u}-\mathbf{p}_{\mathrm{N}}\right\|=\left\|\mathbf{q}_{\mathrm{N}}\right\| .
$$

To construct a solution with higher resolution, we rewrite (10) as

$$
\begin{array}{ll}
\frac{d \mathbf{p}_{N}}{d t}+(\vee \mathbf{A}+\mathbf{C}) \mathbf{p}_{\mathrm{N}}+\Pi_{N} \mathbf{B}\left(\mathbf{p}_{\mathrm{N}}+\mathbf{q}_{\mathrm{N}}, \mathbf{p}_{\mathrm{N}}+\mathbf{q}_{\mathrm{N}}\right)=\Pi_{N} \mathbf{f}, \quad \mathbf{p}_{\mathrm{N}} \in \mathrm{V}_{\mathrm{N}}, \\
\frac{\mathrm{d} \mathbf{q}_{\mathrm{N}}}{\mathrm{dt}}+(\vee \mathbf{A}+\mathbf{C}) \mathbf{q}_{\mathrm{N}}+\mathrm{Q}_{\mathrm{N}} \mathbf{B}\left(\mathbf{p}_{\mathrm{N}}+\mathbf{q}_{\mathrm{N}}, \mathbf{p}_{\mathrm{N}}+\mathbf{q}_{\mathrm{N}}\right)=\mathrm{Q}_{\mathrm{N}} \mathbf{f}, \quad \mathbf{q}_{\mathrm{N}} \in \mathrm{V} \backslash \mathrm{V}_{\mathrm{N}}
\end{array}
$$

The existence of a function $\Phi$ such that $\mathbf{q}_{\mathbf{N}}=\Phi\left(\mathbf{p}_{\mathrm{N}}\right)$ was proven by several researchers (Temam and Wang [9] for example). The graph of $\Phi$ is known as the inertial manifold. The existence of the inertial form suggests that in the first equation (14), $\mathbf{q}_{\mathrm{N}}$ may be replaced by $\Phi\left(\mathbf{p}_{\mathrm{N}}\right)$ to obtain solutions with high resolution, but an analytical or computable form of the exact inertial manifold is not known in general.

The next step is to consider a computable $\Phi_{\text {app }}$ that approximates the exact inertial form $\Phi$. The graph of $\Phi_{\text {app }},\left\{\left(\mathbf{v}, \Phi_{\text {app }}(\mathbf{v})\right) \mid \mathbf{v} \in \mathbf{V}_{N}\right\}$ is known as an approximate inertial manifold. A concrete representation of $\Phi_{\text {app }}$ can be obtained using the observation by Foias et al. [4], that, under certain constraints, in (15), terms containing $\mathbf{q}_{\mathrm{N}}$ other than $(\nu \boldsymbol{A}+\mathbf{C}) \mathbf{q}_{\mathrm{N}}$ can be 
considered negligible compared to other terms in the full system. Hence from (15), the observation leads to an approximation

$$
\Phi_{\text {app }}\left(\mathbf{p}_{\mathrm{N}}\right)=\Phi_{1}\left(\mathbf{p}_{\mathrm{N}}\right):=(\vee \mathbf{A}+\mathbf{C})^{-1} \mathbf{Q}_{\mathrm{N}}\left[\mathbf{f}-\mathbf{B}\left(\mathbf{p}_{\mathrm{N}}, \mathbf{p}_{\mathrm{N}}\right)\right] .
$$

Approximating $Q_{N}$ by $\tilde{Q}_{N}=\Pi_{2 N}-\Pi_{N}$ yields the computable approximation

$$
\tilde{\Phi}_{\text {app }}\left(\mathbf{p}_{\mathrm{N}}\right)=\tilde{\Phi}_{1}\left(\mathbf{p}_{\mathrm{N}}\right):=(\vee \mathbf{A}+\mathbf{C})^{-1} \tilde{Q}_{N}\left[\mathbf{f}-\mathbf{B}\left(\mathbf{p}_{N}, \mathbf{p}_{N}\right)\right] .
$$

The nonlinear Galerkin method is based on (17). More precisely, to obtain nonlinear Galerkin solutions with $2 \mathrm{~N}$ modes, we get a $4\left(\mathrm{~N}^{2}+\mathrm{N}\right)$-dimensional coupled system, with the first $\mathrm{N}^{2}+2 \mathrm{~N}$ equations given by (14) with $\mathbf{q}_{\mathrm{N}}$ replaced by $\tilde{\Phi}_{1}\left(\mathbf{p}_{N}\right)$ and the remaining $3 N^{2}+2 N$ equations for the unknown $\tilde{\Phi}_{1}\left(\mathbf{p}_{N}\right)$ given by (17), leading to the system requiring higher resolution solutions for simulation to move from one time step to the next. Thus each time step requires solutions of elliptic problems on the surface.

A less expensive approach is the post-processing Galerkin method, which was proposed initially for partial differential equations on periodic domains by Archilla et al. [1]. The post-processing algorithm to compute a field $\mathbf{w}_{\mathrm{N}}$ with $2 \mathrm{~N}$ modes, approximating the velocity field $\mathbf{u}$ of (2)-(3) at any fixed time $t^{*}$ is to

1. use a pseudospectral quadrature Galerkin algorithm to compute $\mathbf{u}_{\mathrm{N}}\left(\mathbf{t}^{*}\right)$ in $(12)-(13)$;

2. solve the linear stationary elliptic problem

$$
(v \mathbf{A}+\mathbf{C}) \mathbf{z}_{\mathrm{N}}=\tilde{Q}_{2 \mathrm{~N}}\left\{\mathbf{f}-\mathbf{B}\left(\mathbf{u}_{\mathrm{N}}\left(\mathrm{t}^{*}\right), \mathbf{u}_{\mathrm{N}}\left(\mathrm{t}^{*}\right)\right)\right\}, \quad \tilde{\mathrm{Q}}_{2 \mathrm{~N}}=\Pi_{2 \mathrm{~N}}-\Pi_{\mathrm{N}} ;
$$

3. The new post-processed higher resolution approximation to the velocity field $\mathbf{u}\left(t^{*}\right)$ is $\mathbf{w}_{\mathrm{N}}\left(t^{*}\right):=\mathbf{u}_{\mathrm{N}}\left(\mathrm{t}^{*}\right)+\mathbf{z}_{\mathrm{N}}$.

In the above post-processing algorithm, $2 \mathrm{~N}$ can be replaced by $\mathrm{cN}$, for any integer $c \geq 2$. A complete mathematical analysis of the algorithm, following 
details as described by Archilla et al. [1] and the recent work by Ganesh et al. [5], proving the spectral accuracy of the post-processed solution, is beyond the scope of this article.

\section{Numerical results}

In this section, we simulate (2)-(3) for two classes of the velocity field. The first class of field is induced by a tangential divergence free external force with $2 \mathrm{~N}$ modes (for various choices of $\mathrm{N}$ ) and the force is chosen so that the exact velocity field is known. The benchmark second test case is considered in several references such as $[2,3]$, where the velocity field is generated by a random initial state with fewer modes at $t=0$ than that present in the fields at $t>0$. For both these simulations, the Reynolds number is of the order $v^{-1}=10,000$, where $v$ is the constant viscosity factor in (2).

For the first example, the free external force and initial state in (2)-(3) with $2 \mathrm{~N}$ modes were chosen so that the exact velocity field is

$$
u(t)=\sum_{n=1}^{2 N} g(t)\left[\mathbf{Z}_{n, 0}+2 \sum_{m=1}^{n} \mathfrak{R}\left(\mathbf{Z}_{n, m}\right)\right],
$$

where $\mathfrak{R}(\cdot)$ denotes the real part, and $g(t)$ is the slowly decaying oscillatory function

$$
g(t)=v\left[\frac{g_{1}(t, a, c)}{\left(a^{2}+c^{2}\right)}+\frac{g_{2}(t, b, c)}{\left(b^{2}+c^{2}\right)}\right] \exp (c t), \quad a=5, b=10, c=-0.1,
$$

where

$$
g_{1}(t, a, c)=-a \cos (a t)+c \sin (a t), \quad g_{2}(t, b, c)=c \cos (b t)+b \sin (b t) .
$$

We computed the low frequency solution with $\mathrm{N}$ modes by solving (13) with details given elsewhere [5]. The initial state Galerkin error, before beginning 


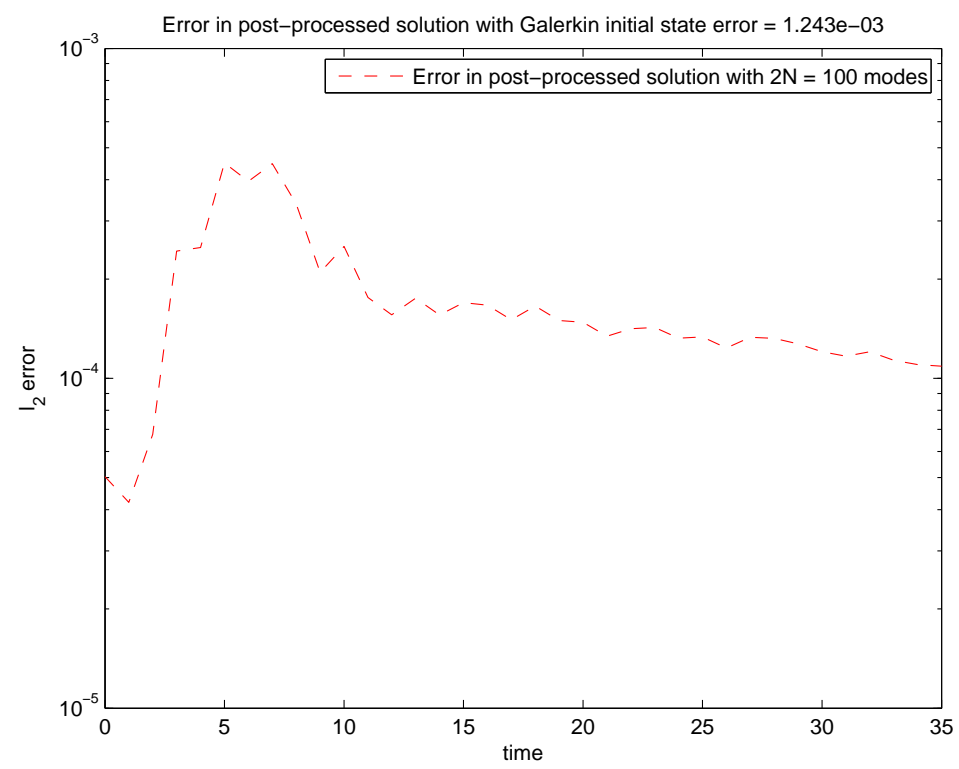

Figure 1: Error in post-processed solution with $2 \mathrm{~N}=100$ and Galerkin initial state error $0.1243 \times 10^{-2}$. 
the adaptive time simulation of (13), is measured by the $l_{2}$-norm of the unused vector with Fourier coefficients of the modes $\mathrm{N}+1$ to $2 \mathrm{~N}$. The adaptive time simulation error tolerance was chosen to be smaller than the initial state Galerkin error. The power of the post-processing approach to approximate the velocity field (18) is demonstrated in Figure 1, where the post-processed solution error for all time is better than the initial Galerkin error with $\mathrm{N}=50$. (We observed similar results for various values of $\mathrm{N}$.)

The second example is (2)-(3) with a random field initial state having 20 modes and the external force being a tangential surface divergence free field with only one non-vanishing time-dependent Fourier coefficient $\widehat{\mathbf{f}(\mathbf{t})}$ 3,0 . These two source fields, given in Figure 2, are similar to those considered by many researchers $[2,3,5]$ and differ mainly by the randomness of the initial state.

For this unknown velocity field test case, as discussed by Debussche et al. [2] and by Ganesh et al. [5], after a sufficient large time, the $n$th mode energy spectrum decays with $\mathrm{O}\left(\mathrm{n}^{-4}\right)$ for $n$ within the inertial range and decays exponentially for $n$ in the dissipation range. Hence in this random initial field test case, the post-processing solution effect is seen mainly at earlier evolution time periods. In particular, the evolution process is such that the initial random flow with several smaller structures will slowly evolve into regular flow with larger structures. Simulation of this flow was carried out first with $\mathrm{N}=50$ and then post-processed to obtain approximate velocity field with $2 \mathrm{~N}$ modes. The simulation results in Figure $3-5$, for $\mathrm{t}=10,20,30$ highlight the power of the post-processing that captures the transition of initial random flow to a regular flow, while the standard Galerkin pseudospectral solution with $\mathrm{N}=50$ leads to regular flow even at $\mathrm{t}=10$.

Acknowledgments The support of the Australian Research Council under its Discovery and Centre of Excellence grant is gratefully acknowledged. We thank the referees for their valuable comments on the article. 


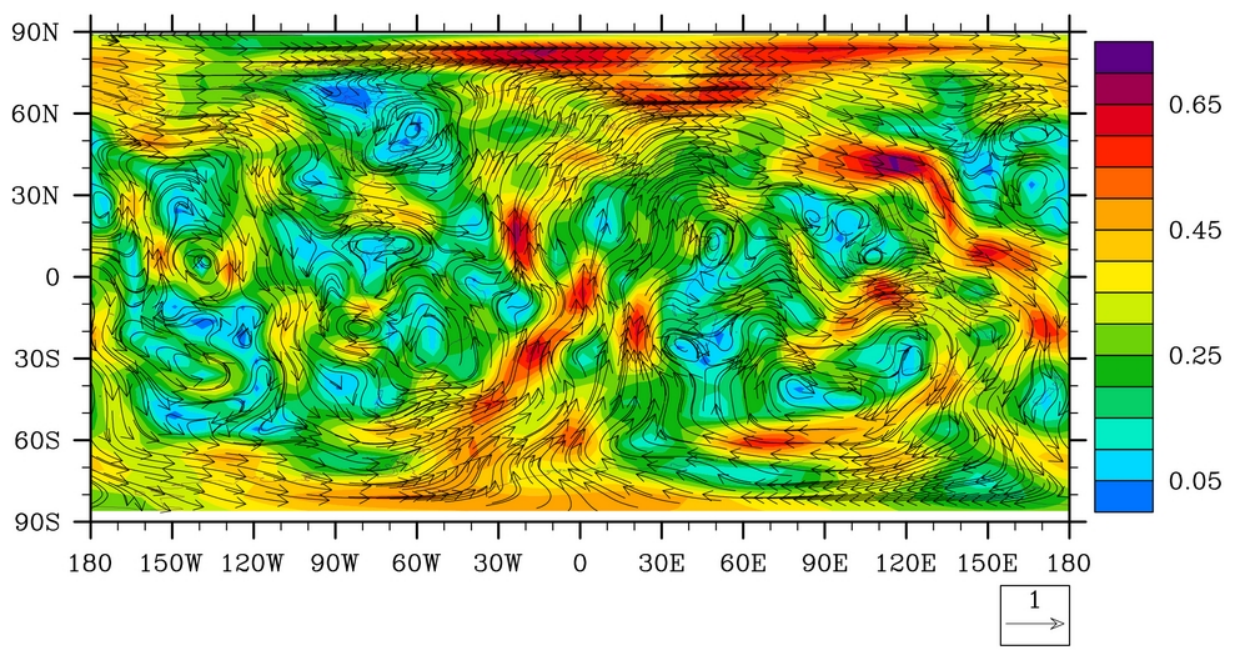

(a)

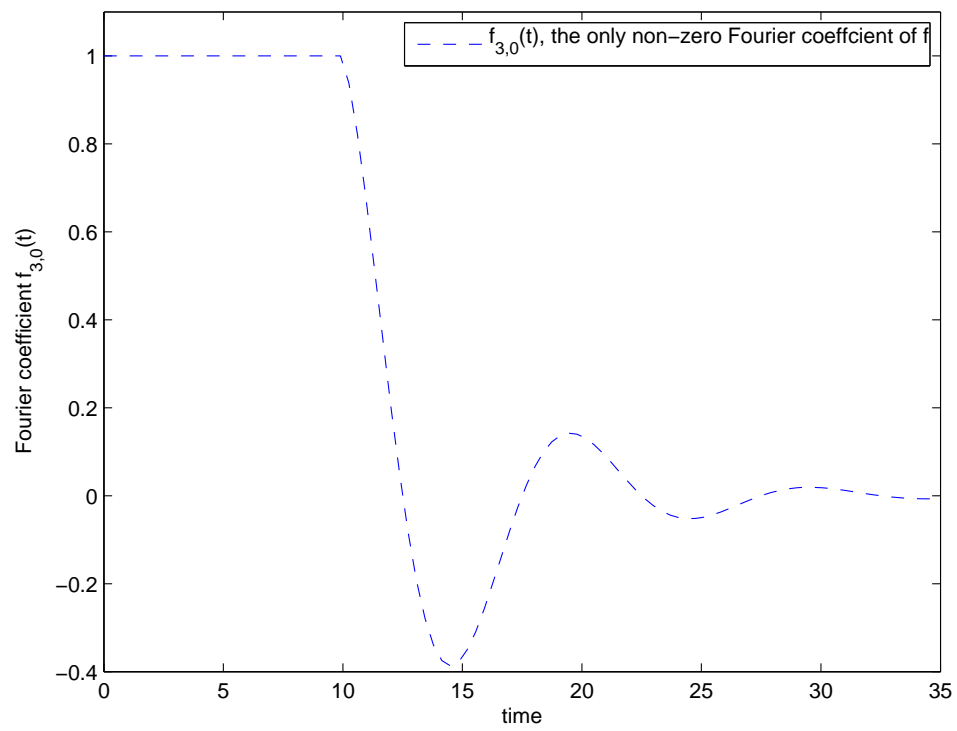

(b)

Figure 2: (a) Random initial state $\mathbf{u}_{0}$; and (b) time dependent external force Fourier coefficient $\widehat{\mathbf{f}(t)}{ }_{3,0}$. 

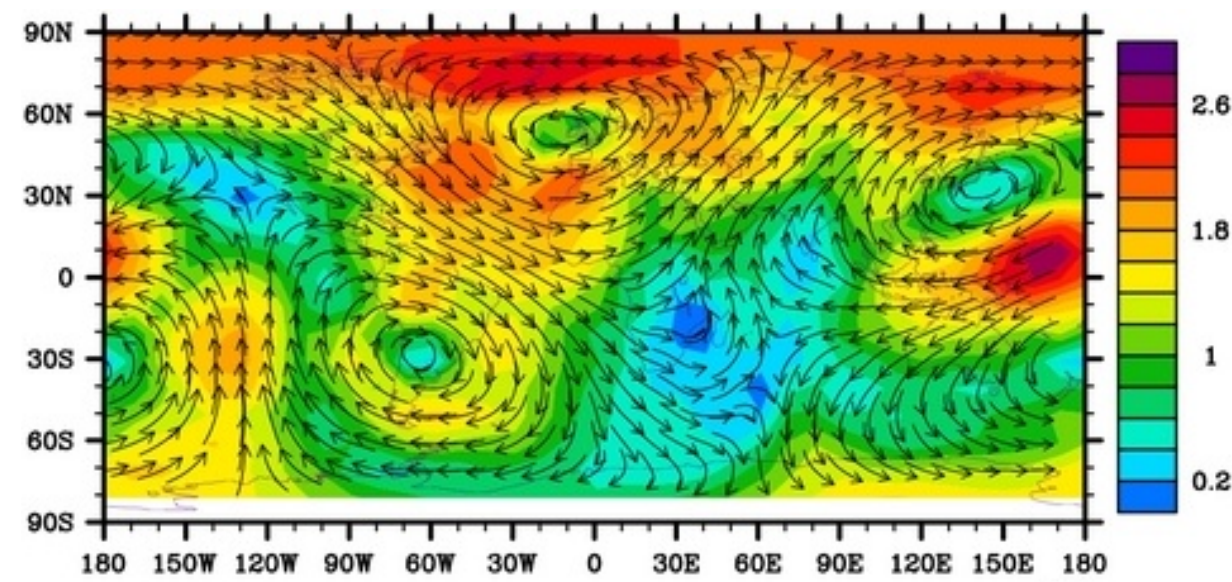

\section{8}
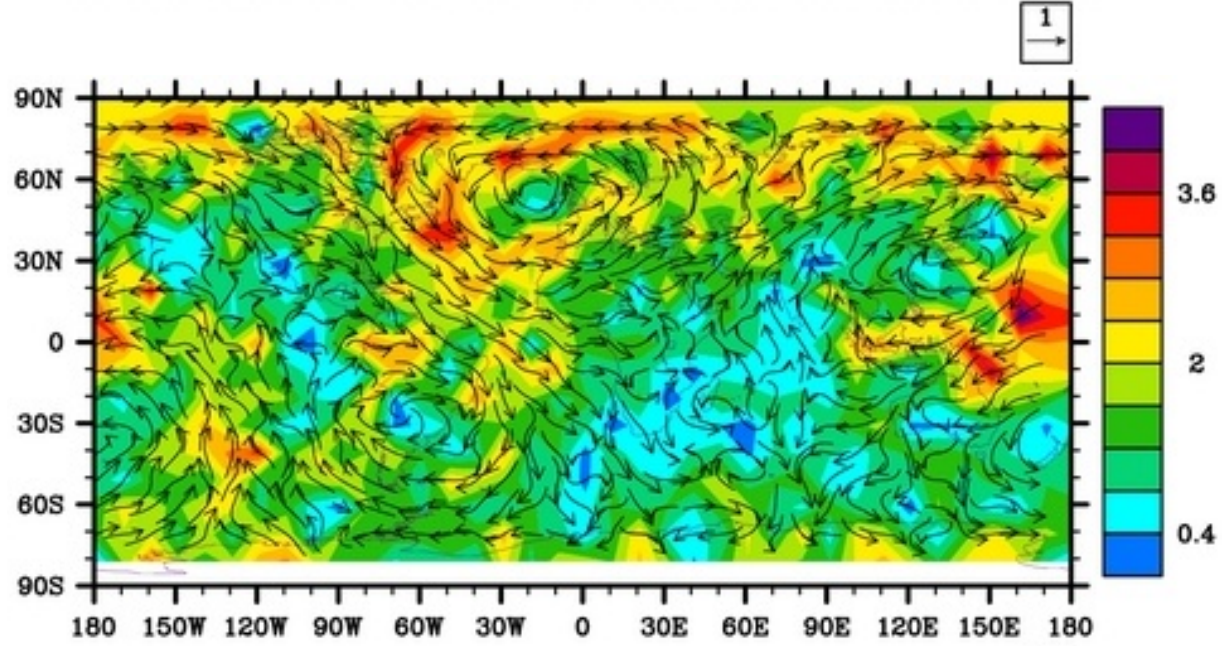

FiguRE 3: Velocity $\mathbf{u}_{50}(\mathbf{t})$ (above) and post-processed velocity (below) at $\mathrm{t}=10$. 

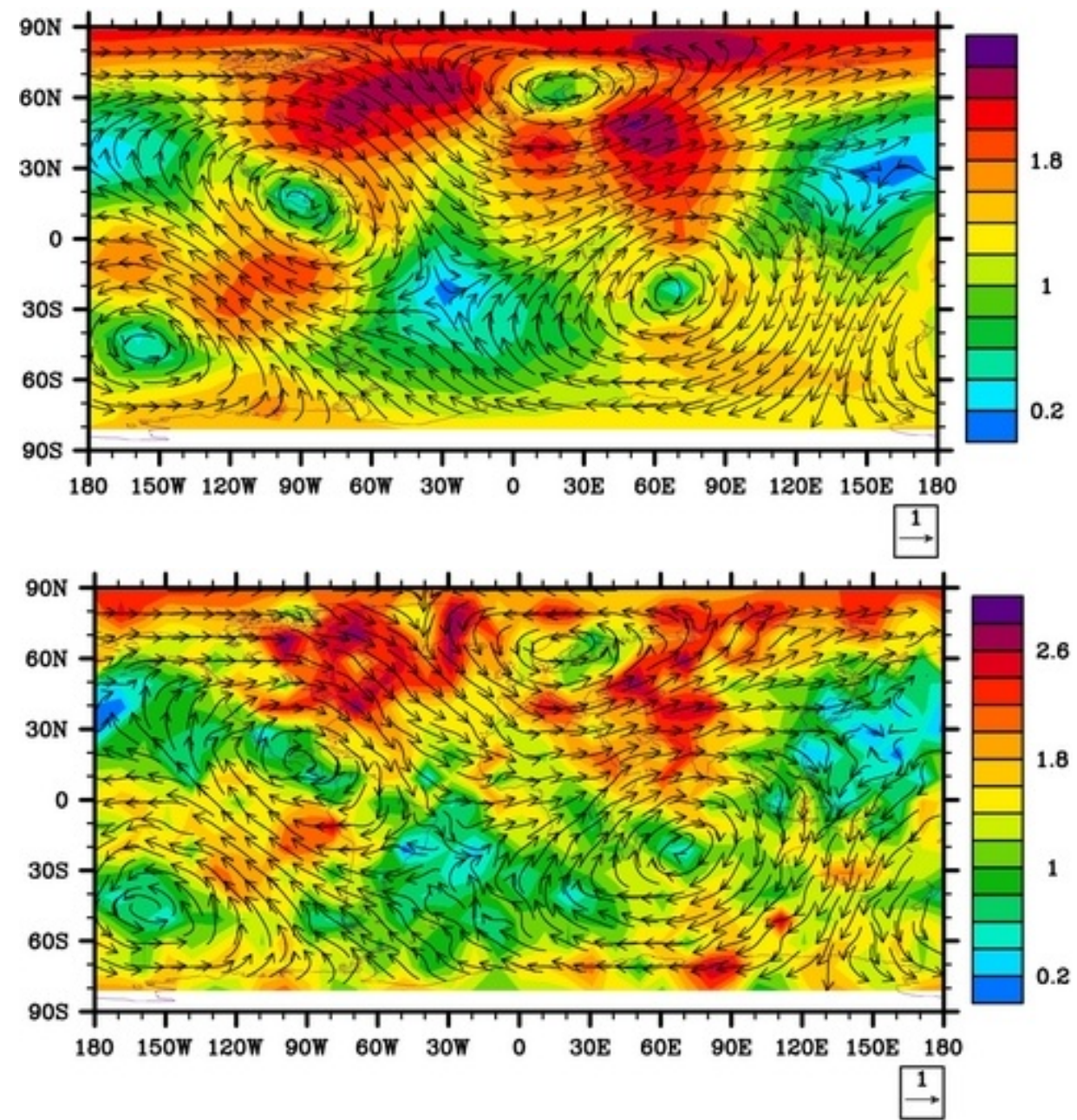

FigURE 4: Velocity $\mathbf{u}_{50}(\mathbf{t})$ (above) and post-processed velocity (below) at $t=20$. 

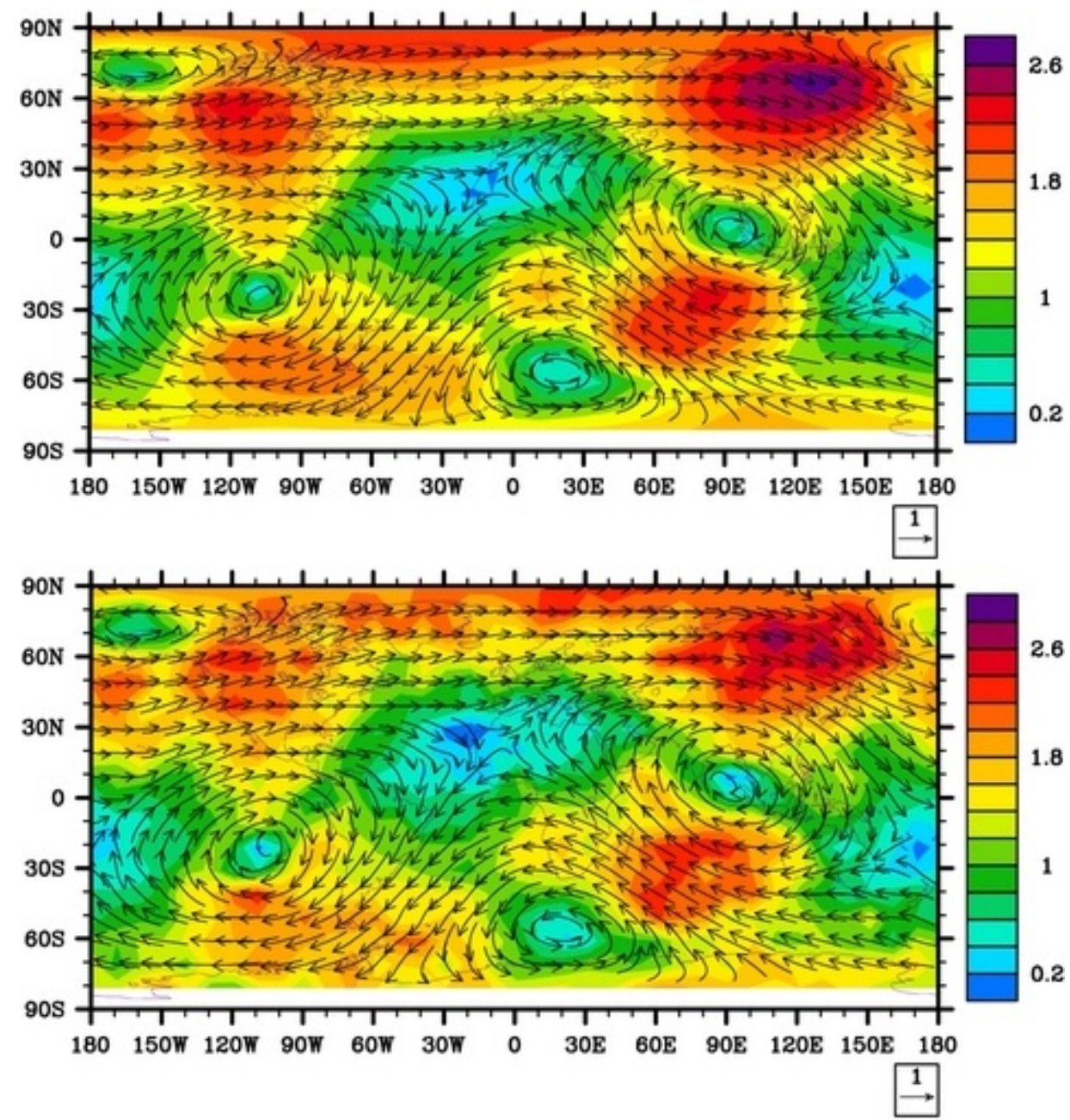

Figure 5: Velocity $\mathbf{u}_{50}(\mathbf{t})$ (above) and post-processed velocity (below) at $\mathrm{t}=30$. 


\section{References}

[1] B. G. Archilla, J. Novo, and E. S. Titi. Postprocessing the Galerkin method: a novel approach to approximate inertial manifolds. SIAM J. Numer. Anal., 35:941-972, 1998. doi:10.1137/S0036142995296096 C93, C97, C98

[2] A. Debussche, T. Dubois, and R. Temam. The nonlinear Galerkin method: a multiscale method applied to the simulation of homogeneous turbulent flows. Theoret. Comput. Fluid Dynamics, 7:279-315, 1995. doi:10.1007/BF00312446 C98, C100

[3] M. J. Fengler and W. Freeden. A nonlinear Galerkin scheme involving vector and tensor spherical harmonics for solving the incompressible Navier-Stokes equation on the sphere. SIAM J. Sci. Comput., 27:967-994, 2004. doi:10.1137/040612567 C91, C92, C93, C98, C100

[4] C. Foias, O. Manley, and R. Temam. Modelling of the interaction of small and large eddies in two dimensional turbulence flows, RAIRO Modél. Math. Anal. Numér., 22:93-118, 1988. C96

[5] M. Ganesh, Q. T. Le Gia, and I. H. Sloan. A pseudospectral quadrature method for Navier-Stokes equations on rotating spheres. Preprint. http://www.mines.edu/ mganesh/NSE-08-pap.pdf C93, C95, C96, C98, C100

[6] A. A. Il'in. The Navier-Stokes and Euler equations on two dimensional closed manifolds, Math. USSR Sbornik, 69:559-579, 1991. doi:10.1070/SM1991v069n02ABEH002116 C93, C94

[7] A. A. Il'in and A. N. Filatov. On unique solvability of the Navier-Stokes equations on the two dimensional sphere. Soviet Math. Dokl., 38:9-13, 1989. C93, C94 
[8] M. Marion and R. Temam. Nonlinear Galerkin methods, SIAM J. Numer. Anal., 26:1139-1157, 1989. doi:10.1137/0726063 C92

[9] R. Temam and S. Wang, Inertial forms of Navier-Stokes equations on the sphere. J. Funct. Anal., 117:215-24, 1993. doi:10.1006/jfan.1993.1126 C93, C96

\section{Author addresses}

1. M. Ganesh, Department of Mathematical and Computer Sciences, Colorado School of Mines, Golden, CO 80401, USA. mailto:mganesh@mines. edu

2. Q. T. Le Gia, School of Mathematics and Statistics, University of New South Wales, Sydney, NSW 2052, Australia.

mailto:mailto:qlegia@unsw. edu.au 